\title{
Clinical Utility of Procalcitonin as a Marker of Sepsis: A Potential Predictor of Causative Pathogens
}

\author{
Atsuko Nakajima ${ }^{1}$, Junko Yazawa ${ }^{1}$, Daisuke Sugiki ${ }^{2}$, Mari Mizuguchi ${ }^{3}$, Hironori Sagara ${ }^{3}$, \\ Masahiro Fujisiro ${ }^{1}$, Mitsuei Shibazaki ${ }^{1}$, Akihiko Hitani ${ }^{1}$, Masako To ${ }^{1}$ and Kosuke Haruki ${ }^{1}$
}

\begin{abstract}
Objective Sepsis is one of the leading causes of mortality in critically ill patients, and providing a timely diagnosis and early intervention is necessary for successful treatment. Procalcitonin (PCT) may be a better marker of sepsis than conventional inflammatory markers. The aim of this study was to evaluate the clinical utility of the PCT level as a marker of sepsis.

Methods Forty-five patients with sepsis, 24 patients with pneumonia who did not meet the SIRS criteria (PN) and 56 controls were enrolled in this study. The levels of PCT and other serum markers were measured, and their utility as markers of sepsis was assessed.

Results The serum PCT levels exhibited statistically significant differences between the three groups $(\mathrm{p}<$ 0.0001). The PCT levels in the sepsis group $(29.3 \pm 85.3 \mathrm{ng} / \mathrm{mL})$ were significantly higher $(\mathrm{p}<0.001)$ than those observed in the PN group $(0.34 \pm 8.6 \mathrm{ng} / \mathrm{mL})$ and the control group $(0.74 \pm 2.1 \mathrm{ng} / \mathrm{mL})$, according to a post hoc analysis. There were no differences in the white blood cell (WBC) counts or C-reactive protein (CRP) levels between the three groups. Fourteen of the 45 patients with sepsis had positive microbiological blood cultures (Gram-positive cocci [GPC] in seven patients, Gram-negative rods [GNR] in six patients, other types of bacteria in one patient). The 13 patients with GNR or GPC were categorized into the GNR group or GPC group according to the identified pathogens. The serum PCT levels were significantly higher in the GNR group $(149.8 \pm 199.7 \mathrm{ng} / \mathrm{mL})$ than in the GPC group $(19.1 \pm 41.8 \mathrm{ng} / \mathrm{mL})(\mathrm{p}<0.05)$, although there were no differences in the WBC counts or CRP levels between these groups. When the cut-off value for the PCT level was set at $16.9 \mathrm{ng} / \mathrm{mL}$, the sensitivity and specificity for the detection of GNR infection were $85.7 \%$ and $83.3 \%$, respectively.

Conclusion The PCT level is a potentially useful marker of the type of causative pathogen in patients with sepsis whose measurement may facilitate the selection of appropriate empiric antibiotic treatment.
\end{abstract}

Key words: procalcitonin, sepsis, bacterial blood culture

(Intern Med 53: 1497-1503, 2014)

(DOI: 10.2169/internalmedicine.53.1785)

\section{Introduction}

Sepsis is one of the leading causes of mortality in critically ill patients in the intensive care unit (1), and providing a timely diagnosis and early intervention for sepsis is important for preventing septic shock and death (2). While microbiological blood cultures are used to identify the causative pathogen of sepsis and help facilitate the selection of antibi- otics, the culture may require several days before the organism is isolated and identified; therefore, culture results may not be sufficiently timely to guide the choice of therapy for sepsis. In addition, microbiological culture exhibits a low sensitivity and specificity for the diagnosis of sepsis (3). Biomarkers of inflammation, such as the white blood cell (WBC) count and C-reactive protein (CRP) level, can also be used to diagnose sepsis, although their specificity is very low. In addition, the WBC count can decrease and the CRP

${ }^{1}$ Department of Laboratory Medicine, Dokkyo Medical University Koshigaya Hospital, Japan, ${ }^{2}$ Shock Trauma Center, Dokkyo Medical University Koshigaya Hospital, Japan and ${ }^{3}$ Department of Respiratory Medicine, Dokkyo Medical University Koshigaya Hospital, Japan Received for publication September 19, 2013; Accepted for publication January 21, 2014 Correspondence to Dr. Kosuke Haruki, kos-h@dokkyomed.ac.jp 
Table 1. Infections in Patients with Sepsis

\begin{tabular}{lr}
\hline Infectious focuses & $\mathrm{n}$ \\
\hline Pneumonia and/or pleural empyema & 10 \\
Meningitis & 4 \\
Microbial infection of burn wounds & 3 \\
Pyelonephritis & 3 \\
Cholangitis & 3 \\
Others & 22 \\
\hline
\end{tabular}

level can remain low in cases of severe sepsis. Therefore, identifying a biomarker with higher sensitivity and specificity for the diagnosis of sepsis would be of benefit.

Procalcitonin (PCT) is a 116-amino acid protein with a molecular mass of $13 \mathrm{kD}$. It is produced by thyroid C cells and converted to calcitonin before being released into the bloodstream. In healthy individuals, PCT is not usually present at a detectable level in the blood. In 1993, a study demonstrated that the serum PCT levels are elevated in patients with sepsis and decrease following successful treatment of sepsis with antibiotics (4). Furthermore, in the context of local bacterial or viral infection, the circulating PCT level remains at $<1.6 \mathrm{ng} / \mathrm{mL}$ (4). Other studies have suggested that measuring the PCT level is useful for making an early diagnosis of sepsis (5), as well as assessing the severity (6) and prognosis $(7,8)$ of septic patients.

On the other hand, the serum PCT level can be affected by lipopolysaccharides, sepsis-related cytokines $(9,10)$ and possibly renal dysfunction (11). The goal of this study was therefore to characterize the clinical utility of the PCT level as a biomarker of sepsis.

\section{Materials and Methods}

\section{Subjects}

We used a prospective observational design for this study. The study population included patients with sepsis, pneumonia without sepsis and a high fever of non-infectious origin. The patients were recruited from the Shock Trauma Center and Department of Respiratory Medicine at Dokkyo Medical University Koshigaya Hospital between May 2010 and December 2010. The patients diagnosed with sepsis had a definitive focus of infection together with two or more systemic inflammatory response syndrome (SIRS) criteria, according to the American College of Chest Physicians/Society of Critical Care Medicine (12). Briefly, these criteria were: 1) a body temperature of $>38^{\circ} \mathrm{C}$ or $<36^{\circ} \mathrm{C} ; 2$ ) a pulse of $>90$ beats $/ \mathrm{min}$; 3) a respiratory rate of $>20$ breaths $/ \mathrm{min}$ or a $\mathrm{PaCO}_{2}$ of $<32 \mathrm{mmHg}$; or 4 ) a WBC count of $>12,000 / \mu \mathrm{L}$ or $<4,000 / \mu \mathrm{L}$. The infectious foci in the patients with sepsis are listed in Table 1. As a local bacterial infection group, patients with pneumonia (diagnosed according to the Japanese Respiratory Society (JRS) Guidelines for the Management of Community-Acquired Pneumonia in Adults) who did not meet the SIRS criteria (12) were recruited. Patients with a high fever $\left(>38.0^{\circ} \mathrm{C}\right)$ of non-infectious origin were
Table 2. Comorbidities of Patients with Sepsis, of Patients with Pneumonia without Sepsis, and of Control Subjects

\begin{tabular}{lr}
\hline & $\mathrm{n}$ \\
\hline Patients with sepsis & \\
Abdominal trauma & 7 \\
Cerebrovascular disease & 6 \\
Fracture & 5 \\
Diabetes mellitus & 5 \\
Heatstroke & 4 \\
Heart failure & 4 \\
Burn & 3 \\
Acute respiratory failure & 2 \\
Acute renal failure & 1 \\
Others & 8 \\
\hline Patients with pneumonia without sepsis & \\
Lung cancer & 6 \\
Renal failure & 1 \\
Cardiovascular diseases & 1 \\
None & 16 \\
\hline Control subjects & 9 \\
Gastrointestinal diseases & 8 \\
Heart failure & 8 \\
Postoperative complication (fever) & 6 \\
Cerebrovascular disease & 3 \\
Renal failure & 3 \\
Leukemia & 19 \\
Others &
\end{tabular}

enrolled in this study as controls. The comorbidities of the three groups of subjects are listed in Table 2.

Phlebotomy for the measurement of the levels of PCT and other serum markers was performed at the time of diagnosis of sepsis and pneumonia in the sepsis group and pneumonia group, respectively. Phlebotomy was performed in the controls at the time of a high fever.

This study was reviewed and approved by the Dokkyo Medical University Koshigaya Hospital Research Ethics Committee and conducted in accordance with the Helsinki Declaration.

\section{PCT measurements}

The PCT levels were measured via an electrochemiluminescent immunoassay using Elecsys reagent, Elecsys BRAHMS PCT and Cobas e411 (Roche Diagnostics, Tokyo, Japan), according to the manufacturer's instructions. The lower limit of detection was $0.02 \mathrm{ng} / \mathrm{mL}$. The CRP levels were measured according to the latex agglutination method using Nanopia CRP reagent (Sekisui Medical Co., Ltd., Tokyo, Japan) and JCA-BM2250 (JEOL, Ltd., Tokyo, Japan). The WBC counts were measured using flow cytometry with XE5000 (Sysmex Corporation, Tokyo, Japan). The endotoxin levels were measured via a turbidimetric time assay, which was performed by a clinical laboratory testing company (SRL, Inc., Tokyo, Japan). Blood sampling for the microbiological blood culture was performed prior to the administration of antibiotics, and two sets of samples were obtained from each patient. Identical bacteria detected from both sets of samples were defined as causative pathogens.

\section{Statistics}

The results are presented as the mean \pm standard deviation 
A

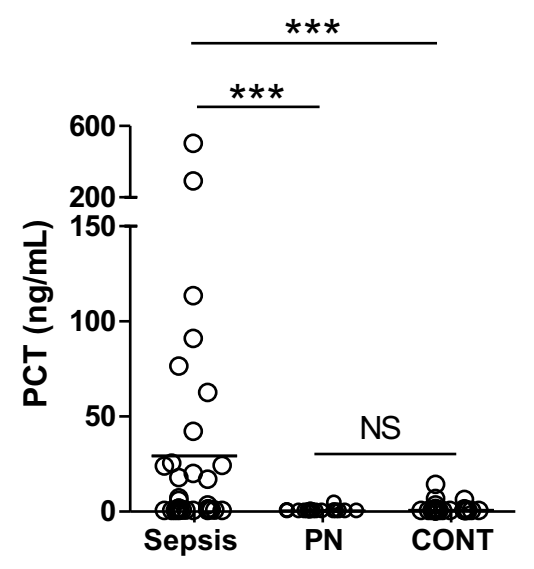

C

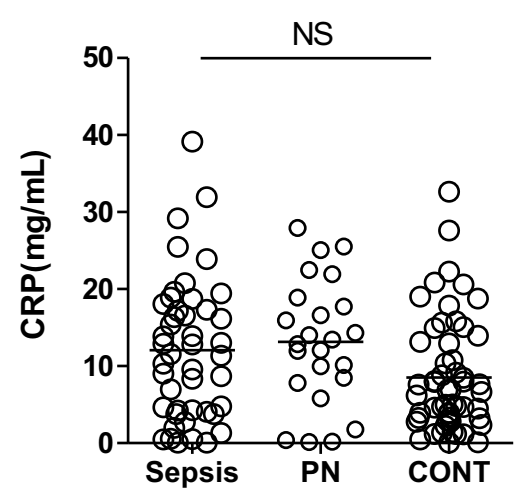

B

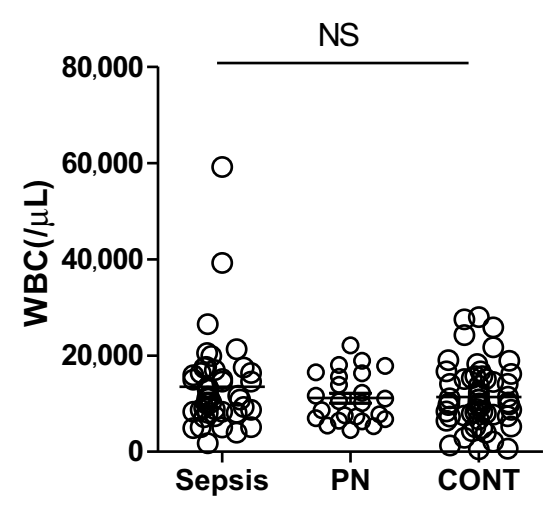

$\mathrm{D}$

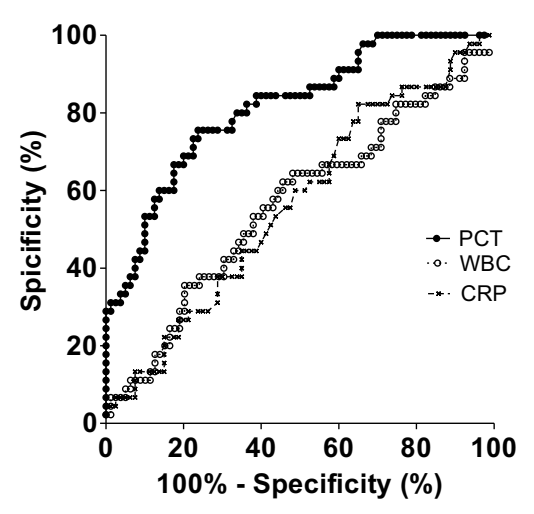

Figure 1. The serum PCT levels (A), WBC counts (B) and CRP levels (C) in the sepsis group, PN group and control group. A receiver operating analysis of the serum PCT levels, WBC counts and CRP levels in the patients with and without sepsis (control and PN groups) was performed (D). ***: p<0.001; NS: not significant, PCT: procalcitonin, WBC: white blood cell, CRP: C-reactive protein, PN: pneumonia who did not meet the SIRS criteria

of the mean. Comparisons between two groups of data were made using the Mann-Whitney $U$ test. Comparisons between three groups of data were made using the Kruskal-Wallis test, followed by a post hoc test. Correlations between two groups of data were analyzed using Spearman's rank correlation coefficient. A receiver operating characteristic (ROC) analysis was used to evaluate the results of diagnostic tests. A $\mathrm{p}$ value of less than 0.05 was considered to indicate statistical significance.

The statistical analyses were performed using the IBM SPSS Statistics version 20 (IBM, Armonk, USA) and Graph Pad Prism 5 (Graph Pad Prism, San Diego, USA) software programs.

\section{Results}

\section{PCT as a diagnostic biomarker of sepsis}

Forty-five patients with sepsis (29 men and 16 women; age, $57.2 \pm 17.7$ years), 24 patients with pneumonia who did not meet the SIRS criteria (PN) (15 men and nine women; age, 70.5 \pm 9.8 years) and 56 control patients (43 men and 13 women; age, $62 \pm 18.3$ years) were enrolled in this study. The serum PCT levels exhibited statistically significant differences between the three groups $(\mathrm{p}<0.0001)$. Post hoc testing demonstrated that the PCT levels in the sepsis group (29.3 \pm $85.3 \mathrm{ng} / \mathrm{mL})$ were significantly higher $(\mathrm{p}<0.001)$ than those observed in the PN group $(0.34 \pm 8.6 \mathrm{ng} / \mathrm{mL})$ and the control group $(0.74 \pm 2.1 \mathrm{ng} / \mathrm{mL})$ (Fig. 1A). There were no differences in the WBC counts or CRP levels between the three groups (WBC, $13,613 \pm 9,681 / \mu \mathrm{L}$ in the sepsis group, $11,146 \pm 5,088 / \mu \mathrm{L}$ in the PN group and $11,352 \pm 6,375 / \mu \mathrm{L}$ in the control group, $\mathrm{p}=0.49$; CRP, $12.3 \pm 9.0 \mathrm{mg} / \mathrm{dL}$ in the sepsis group, $12.5 \pm 7.6 \mathrm{mg} / \mathrm{dL}$ in the $\mathrm{PN}$ group and $8.54 \pm 7.3$ $\mathrm{mg} / \mathrm{dL}$ in the control group, $\mathrm{p}=0.052$ ) (Fig. 1B, C). A ROC analysis was used to evaluate the utility of the markers for diagnosing sepsis (sepsis group vs. PN group plus control group). The ROC analysis showed an area under the curve (AUC) of 0.80 for the PCT level, 0.56 for the WBC count and 0.60 for the CRP level (Fig. 1D). The sensitivity and specificity of the PCT level for the diagnosis of sepsis were $57.6 \%$ and $87.5 \%$, respectively, when the cut-off value was 
set at $0.5 \mathrm{ng} / \mathrm{mL}$. In contrast, the sensitivity and specificity of the WBC count for the diagnosis of sepsis were $97.8 \%$ and $8.9 \%$, respectively, when the cut-off value was set at $3,500 / \mu \mathrm{L}$. The sensitivity and specificity of the CRP level for the diagnosis of sepsis were $95.6 \%$ and $5.1 \%$, respectively, when the cut-off value was set at $0.3 \mathrm{mg} / \mathrm{dL}$.

\section{Association between the PCT level and microbio- logical blood culture results}

Fourteen of the $45(31.1 \%)$ patients in the sepsis group had positive microbiological blood culture results, while 27 of the $45(60.0 \%)$ patients had positive PCT results when the cut-off value was set at $0.5 \mathrm{ng} / \mathrm{mL}$ (Table 3 ). The rate of positive concordance between the results of the microbiological blood cultures and the PCT tests in the culturepositive patients was $85.7 \%$, while that of negative concordance in the culture-negative patients was $51.6 \%$.

The details of each patient with positive microbiological blood culture results are presented in Table 4. Gram-positive cocci (GPC) were identified in the blood samples of seven patients, Gram-negative rods (GNR) were identified in the blood samples of six patients, and Gram-positive rods were identified in the blood samples of one patient. The 13 patients with either GPC or GNR infection were divided into a GNR group and a GPC group according to the bacteria identified in the blood samples. The serum PCT levels in the GNR group $(149.8 \pm 199.7 \mathrm{ng} / \mathrm{mL})$ were significantly higher

Table 3. Association between the Results of Microbiological Blood Culture and the PCT Test

\begin{tabular}{llrrr}
\hline & & \multicolumn{2}{l}{ PCT } & \multicolumn{2}{c}{ Total } \\
& & Positive & Negative & \\
\hline Culture & Positive & 12 & 2 & 14 \\
results & Negative & 15 & 16 & 31 \\
\hline Total & & 27 & 18 & 45 \\
\hline PCT: procalcitonin & & & &
\end{tabular}

$(p=0.035)$ than those observed in the GPC group $(19.1 \pm 41.8$ $\mathrm{ng} / \mathrm{mL}$ ) (Fig. 2A); however, there were no differences in the WBC counts or CRP levels between the two groups (WBC, $20,033 \pm 20,877 / \mu \mathrm{L}$ in the GNR group and $12,086 \pm 4,317 / \mu \mathrm{L}$ in the GPC group, $\mathrm{p}=0.83$; CRP, $14.3 \pm 7.6 \mathrm{mg} / \mathrm{dL}$ in the GNR group and $9.1 \pm 7.9 \mathrm{mg} / \mathrm{dL}$ in the GPC group, $\mathrm{p}=0.18$ ) (Fig. 2B, C). A ROC analysis showed an AUC value of 0.85 for the PCT level, 0.54 for the WBC count and 0.73 for the CRP level (Fig. 2D). When the cut-off value for the PCT level was set at $16.9 \mathrm{ng} / \mathrm{mL}$, the sensitivity, specificity, positive predictive value and negative predictive value for GNR were $85.7 \%, 83.3 \%, 85 \%$ and $83 \%$, respectively. Regarding the association between the endotoxin and PCT levels, the endotoxin-positive $(0.8 \mathrm{pg} / \mathrm{mL}$ or higher $)$ group had significantly higher PCT levels than the endotoxin-negative (less than $0.8 \mathrm{pg} / \mathrm{mL}$ ) group (Fig. 2E).

\section{Discussion}

This study showed the PCT level, but not WBC count or CRP level, to be significantly higher in the sepsis group than in the control and PN groups. Furthermore, the ROC analysis showed an AUC for the PCT level of 0.80, suggesting that the PCT test had moderate accuracy (according to the classification of Akobeng (13)) for diagnosing sepsis. This finding is consistent with the observations of previous reports suggesting that the PCT level is superior to the endotoxin, interleukin (IL)-6 and CRP levels as a biomarker for the diagnosis of sepsis $(5,8,14-19)$. In addition to the confirmation study, the present study provides novel evidence that: 1) the serum PTC level is a superior marker of sepsis, but not local infections, such as pneumonia, and 2) serum PCT elevation is more closely associated with GNR infection than GPC infection in patients with sepsis.

In this study, there were no significant differences in the PCT levels between the PN group and the control group.

Table 4. Details of Each Patient with Sepsis with Positive Microbiological Blood Culture Results

\begin{tabular}{|c|c|c|c|c|c|c|c|c|c|c|}
\hline $\mathrm{Pt}$ & $\begin{array}{l}\text { Age } \\
\text { (years) }\end{array}$ & Sex & Main Diseases & APACHE & $\begin{array}{l}\text { PCT } \\
(\mathrm{ng} / \mathrm{mL})\end{array}$ & $\begin{array}{l}\text { WBC } \\
(/ \mu \mathrm{L})\end{array}$ & $\begin{array}{l}\text { CRP } \\
(\mathrm{mg} / \mathrm{dL})\end{array}$ & $\begin{array}{l}\text { Endotoxin } \\
(\mathrm{pg} / \mathrm{mL})\end{array}$ & Bacteria & Outcome \\
\hline 1 & 61 & M & Mud water aspiration, ARDS & 20 & 285.0 & 1,800 & 4.8 & 513 & P. aeruginosa, E.coli & Dead \\
\hline 2 & 62 & M & Septic shock & 23 & 496.1 & 26,500 & 18.9 & 185 & Klebsiella sp & Dead \\
\hline 3 & 77 & M & Pneumonia & 26 & 0.7 & 8,800 & 8.3 & $<0.8$ & E.cloacae & Dead \\
\hline 4 & 74 & M & Pyelonephritis & 23 & 17.3 & 9,300 & 25.5 & $<0.8$ & E. aerogenes & Survived \\
\hline 5 & 59 & M & Hematemesis & 16 & 23.7 & 14,600 & 17.2 & $<0.8$ & E.coli & Survived \\
\hline 6 & 79 & M & Cholangitis & 42 & 75.9 & 59,200 & 11.6 & 7.4 & E.coli & Dead \\
\hline 7 & 58 & $\mathrm{~F}$ & Bacterial meningitis & 18 & 16.5 & 8,100 & 16.3 & $<0.8$ & S.pneumoniae & Dead \\
\hline 8 & 42 & M & Ventricular fibrillation & 31 & 0.5 & 11,400 & 4.7 & $<0.8$ & S.epidermidis & Survived \\
\hline 9 & 61 & M & Traffic injury & 31 & 3.0 & 19,900 & 16.1 & $<0.8$ & S.epidermidis & Survived \\
\hline 10 & 57 & $\mathrm{~F}$ & Diabetic acidosis & 35 & 0.5 & 11,000 & 3.8 & $<0.8$ & Streptococcus sp. & Survived \\
\hline 11 & 51 & $\mathrm{~F}$ & Aspiration pneumonia & 12 & 113.0 & 15,900 & 19.7 & $<0.8$ & Peptostrep.micros & Survived \\
\hline 12 & 50 & M & Hyperammonemia, liver damage & 13 & 0.1 & 10,000 & 0.5 & $<0.8$ & S.epidermidis & Survived \\
\hline 13 & 73 & M & Traffic injury, Cerebral hematoma & 21 & 0.1 & 8,300 & 2.7 & $<0.8$ & S.epidermidis & Survived \\
\hline 14 & 70 & M & Cerebrovascular disease & 32 & 0.7 & 16,400 & 13.8 & $<0.8$ & B.cereus & Dead \\
\hline
\end{tabular}

APACHE: Acute Physiology and Chronic Health Evaluation, PCT: procalcitonin, WBC: white blood cell, CRP: C-reactive protein, P. aeruginosa: Pseudomonas aeruginosa, E. coli: Escherichia coli, E. cloacae: Enterobacter cloacae, E. aerogenes: Enterobacter aerogenes, S. pneumoniae: Streptococcus pneumonia, S. epidermidis: Staphylococcus epidermidis, Peptostrep. micros: Peptostreptococcus micros, B. cereus: Bacillus cereus 
A

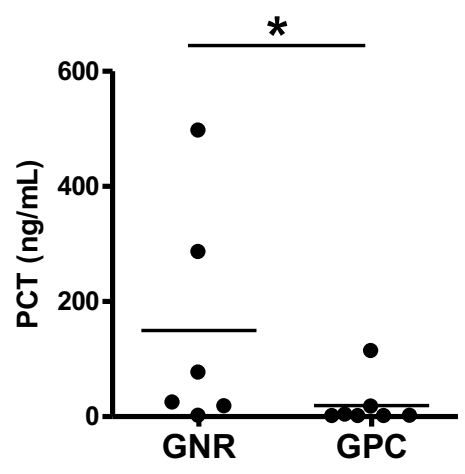

B

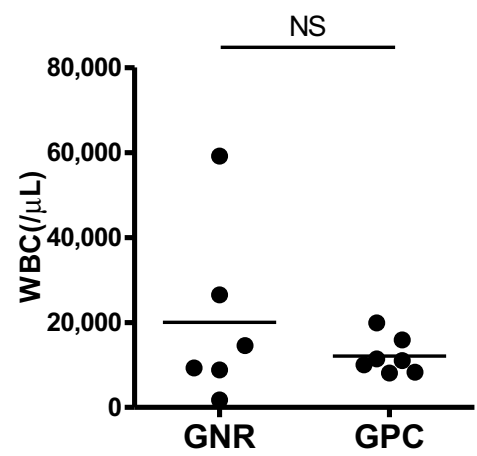

C

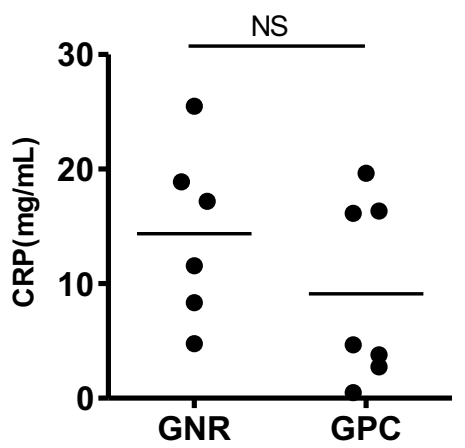

D

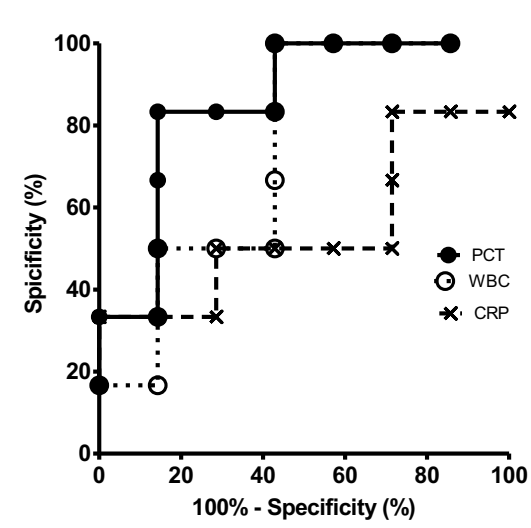

$\mathrm{E}$

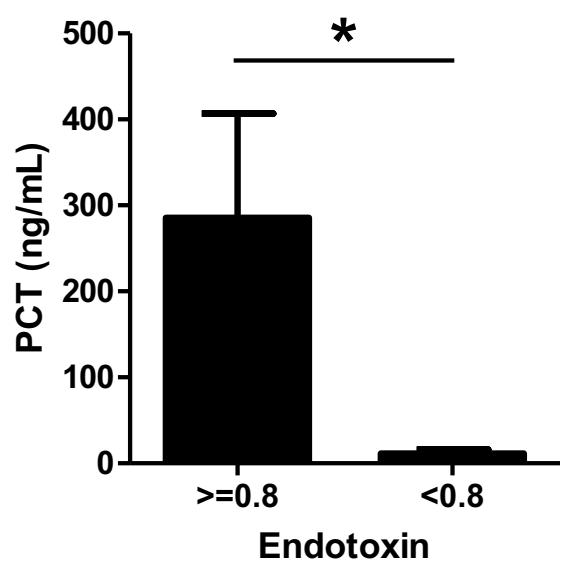

Figure 2. Thirteen patients were categorized into one of two groups according to the pathogens identified in blood cultures (GNR or GPC), and their serum markers were compared. The PCT levels (A), WBC counts (B) and CRP levels (C). A receiver operating analysis of the serum PCT levels, WBC counts and CRP levels in the two groups of patients (D). The 13 patients were also categorized into two groups according to the endotoxin level (cut-off value: $0.8 \mathrm{pg} / \mathrm{mL}$ ), and the PCT levels were compared between the two groups (E). *: p < 0.05; NS: not significant, GNR: Gram-negative rods, GPC: Gram-positive cocci, PCT: procalcitonin, WBC: white blood cell, CRP: C-reactive protein

This finding suggests that the PCT level may not be a good diagnostic marker of local infection, such as pneumonia. In fact, only $34.1 \%$ of the patients with pneumonia exhibited positive PCT levels (more than $0.5 \mathrm{ng} / \mathrm{mL}$ ) (20), although it was not clear whether these patients had sepsis in addition to pneumonia. Furthermore, $93.3 \%$ of the patients with mild community-acquired pneumonia had a negative PCT level (20), suggesting that the sensitivity of this parameter is insufficient for diagnosing pneumonia. Therefore, the PCT level may be a marker of systemic inflammation, but not a diagnostic marker for local infection in patients without SIRS.

The rate of positive concordance between the results of the microbiological blood cultures and PCT tests was $85.7 \%$, while that of negative concordance was $51.6 \%$. This finding is likely related to the low sensitivity of blood cultures for the diagnosis of sepsis $(31.1 \%)$ and is consistent with the results of previous reports showing that the sensitivity of microbiological blood cultures is low, even in patients with severe sepsis (3). However, the rate of positive concordance between the results of the microbiological blood cultures and PCT tests in the culture-positive patients was fairly high; therefore, we compared the PCT levels between the patients with GNR infection and those with GPC infection. Interestingly, the PCT levels were significantly higher in the GNR group than in the GPC group, and the ROC analysis suggested that the PCT test had moderate accuracy (according to the classification of Akobeng (13)) for predicting the type of causative bacteria of sepsis (i.e., GPC vs. GNR). When the cut-off value for the PCT level was set at $16.9 \mathrm{ng} / \mathrm{mL}$, good sensitivity and specificity for predicting the type of causative pathogen were observed. These findings suggest that the PCT level may be of use in predicting the type of causative bacteria in patients with sepsis, even 
before definitive blood culture results become available.

In regards to the difference in the PCT levels observed between the GPC and GNR groups, the potential for contamination from the skin surface should be considered in the GPC group, as GPC is a major component of the normal skin flora. However, two sets of samples for microbiological blood culture were obtained from each patient, and identical bacteria detected in both sets were defined as causative pathogens in this study. Therefore, the possibility of contamination as a confounding factor is negligible. The mechanisms underlying the higher PCT levels noted in the GNR group relative to the GPC group remain unknown; however, the higher PCT levels observed in the GNR group may be associated with the elevated serum endotoxin levels measured in this group, as the endotoxin levels were not detectable in the GPC group, although a level of $>0.8 \mathrm{pg} / \mathrm{mL}$ was observed in three of six patients with a high PCT level in the GNR group. A previous in vitro study also demonstrated that lipopolysaccharide, a major component of endotoxin, stimulates the PCT expression in isolated peripheral blood mononuclear cells (10). Therefore, the PCT level may also be a useful surrogate marker of the serum endotoxin concentration, which otherwise requires a very specialized assay to measure.

Another important clinical characteristic of PCT is the more dynamic change observed in the PCT level in response to changes in the patient's clinical state compared with the CRP level. In a previous study, there was a marked difference in kinetics between PCT and CRP under iatrogenic sepsis: the PTC level was detectable 2.5 hours after the injection of contaminated medicine, reaching a peak at 13.5 hours after injection, while the CRP level reached a peak 30 hours after injection (21). In addition, the half-life of serum PCT is shorter than that of serum CRP (22). This quality may enable the PCT level to be used to make an earlier diagnosis of sepsis and may also aid in decision-making regarding the timing of the initiation of empiric antibiotics and the discontinuation of antibiotic therapy (22-24). Furthermore, the present study showed that measuring the PCT level may help to raise the index of suspicion for Grampositive vs. Gram-negative organisms as the causative pathogen, which further increases its utility in the selection of specific antibiotic regimens.

One limitation of this study is the small number of patients recruited, especially with respect to the analysis of the association between the results of the microbiological blood cultures (GPC vs. GNR) and the PCT tests. Further investigation using a larger scale study is needed to confirm our results and the diagnostic potential of the PCT level.

In conclusion, the present data demonstrate that the PCT level is a useful marker of the type of causative pathogen in patients with sepsis whose measurement may facilitate the selection of appropriate empiric antibiotic therapy.

The authors state that they have no Conflict of Interest (COI).

\section{Acknowledgement}

The authors thank Dr. Keiichi Ikegami for his supervision of the recruitment of patients with sepsis for this study.

\section{References}

1. Parrillo JE, Parker MM, Natanson C, et al. Septic shock in humans. Advances in the understanding of pathogenesis, cardiovascular dysfunction, and therapy. Ann Intern Med 113: 227-242, 1990.

2. Garnacho-Montero J, Garcia-Garmendia JL, Barrero-Almodovar A, Jimenez-Jimenez FJ, Perez-Paredes C, Ortiz-Leyba C. Impact of adequate empirical antibiotic therapy on the outcome of patients admitted to the intensive care unit with sepsis. Crit Care Med 31: 2742-2751, 2003.

3. Tang BM, Eslick GD, Craig JC, McLean AS. Accuracy of procalcitonin for sepsis diagnosis in critically ill patients: systematic review and meta-analysis. Lancet Infect Dis 7: 210-217, 2007.

4. Assicot M, Gendrel D, Carsin H, Raymond J, Guilbaud J, Bohuon C. High serum procalcitonin concentrations in patients with sepsis and infection. Lancet 341: 515-518, 1993.

5. Wacker C, Prkno A, Brunkhorst FM, Schlattmann P. Procalcitonin as a diagnostic marker for sepsis: a systematic review and metaanalysis. Lancet Infect Dis 13: 426-435, 2013.

6. Endo S, Kasai T, Inada K. Evaluation of procalcitonin levels in patients with systemic inflammatory response syndrome as the diagnosis of infection and the severity of illness. Kansenshogaku Zasshi (The Journal of the Japanese Association for Infectious Diseases) 73: 197-204, 1999 (in Japanese, Abstract in English).

7. Jensen JU, Heslet L, Jensen TH, Espersen K, Steffensen P, Tvede M. Procalcitonin increase in early identification of critically ill patients at high risk of mortality. Crit Care Med 34: 2596-2602, 2006.

8. Theodorou VP, Papaioannou VE, Tripsianis GA, et al. Procalcitonin and procalcitonin kinetics for diagnosis and prognosis of intravascular catheter-related bloodstream infections in selected critically ill patients: a prospective observational study. BMC Infect Dis 12: 247, 2012.

9. Becker KL, Snider R, Nylen ES. Procalcitonin assay in systemic inflammation, infection, and sepsis: clinical utility and limitations. Crit Care Med 36: 941-952, 2008.

10. Oberhoffer M, Stonans I, Russwurm S, et al. Procalcitonin expression in human peripheral blood mononuclear cells and its modulation by lipopolysaccharides and sepsis-related cytokines in vitro. $\mathbf{J}$ Lab Clin Med 134: 49-55, 1999.

11. Kapoor S. Procalcitonin and its utility as a diagnostic and prognostic assay in patients with renal dysfunction and immunosuppressive conditions. J Infect Chemother 15: 58, 2009.

12. No authors listed. American College of Chest Physicians/Society of Critical Care Medicine Consensus Conference: definitions for sepsis and organ failure and guidelines for the use of innovative therapies in sepsis. Crit Care Med 20: 864-874, 1992.

13. Akobeng $\mathrm{AK}$. Understanding diagnostic tests 3 : receiver operating characteristic curves. Acta Paediatr 96: 644-647, 2007.

14. Aikawa N, Fujishima $S$, Endo $S$, et al. Multicenter prospective study of procalcitonin as an indicator of sepsis. J Infect Chemother 11: 152-159, 2005.

15. Buhaescu I, Yood RA, Izzedine H. Serum procalcitonin in systemic autoimmune diseases: where are we now? Semin Arthritis Rheum 40: 176-183, 2010.

16. Castelli GP, Pognani C, Meisner M, Stuani A, Bellomi D, Sgarbi L. Procalcitonin and C-reactive protein during systemic inflammatory response syndrome, sepsis and organ dysfunction. Crit Care 8: R234-R242, 2004.

17. Gibot S, Bene MC, Noel R, et al. Combination biomarkers to diagnose sepsis in the critically ill patient. Am J Respir Crit Care 
Med 186: 65-71, 2012.

18. Jekarl DW, Lee SY, Lee J, et al. Procalcitonin as a diagnostic marker and IL-6 as a prognostic marker for sepsis. Diagn Microbiol Infect Dis 75: 342-347, 2013.

19. Tamaki K, Kogata Y, Sugiyama D, et al. Diagnostic accuracy of serum procalcitonin concentrations for detecting systemic bacterial infection in patients with systemic autoimmune diseases. J Rheumatol 35: 114-119, 2008.

20. Hirakata Y, Yanagihara K, Kurihara S, et al. Comparison of usefulness of plasma procalcitonin and C-reactive protein measurements for estimation of severity in adults with communityacquired pneumonia. Diagn Microbiol Infect Dis 61: 170-174, 2008.
21. Brunkhorst FM, Heinz U, Forycki ZF. Kinetics of procalcitonin in iatrogenic sepsis. Intensive Care Med 24: 888-889, 1998.

22. Kaur K, Mahajan R, Tanwar A. A novel marker procalcitonin may help stem the antibiotic overuse in emergency setting. Int $\mathrm{J}$ Appl Basic Med Res 3: 77-83, 2013.

23. Hohn A, Schroeder S, Gehrt A, et al. Procalcitonin-guided algorithm to reduce length of antibiotic therapy in patients with severe sepsis and septic shock. BMC Infect Dis 13: 158, 2013.

24. Schuetz P, Raad I, Amin DN. Using procalcitonin-guided algorithms to improve antimicrobial therapy in ICU patients with respiratory infections and sepsis. Curr Opin Crit Care 19: 453-460, 2013.

(C) 2014 The Japanese Society of Internal Medicine http://www.naika.or.jp/imonline/index.html 